\title{
SPECTRAL PROPERTIES OF ELEMENTARY OPERATORS. II BY
}

\author{
LAWRENCE A. FIALKOW ${ }^{1}$
}

\begin{abstract}
Let $A=\left(A_{1}, \ldots, A_{n}\right)$ and $B=\left(B_{1}, \ldots, B_{n}\right)$ denote commutative $n$-tuples of operators on a Hilbert space $\not$. Let $R_{A B}$ denote the elementary operator on $\mathcal{L}(\mathcal{K})$ defined by $R_{A B}(X)=A_{1} X B_{1}+\cdots+A_{n} X B_{n}$. We obtain new expressions for the essential spectra of $R_{A B}$ and $R_{A B} \mid J$ (the restriction of $R_{A B}$ to a norm ideal $J$ of $\mathcal{L}(\mathcal{H})$ ). We also study isolated points of joint spectra defined in the sense of $R$. Harte.
\end{abstract}

1. Introduction. Let $\not$ denote an infinite-dimensional Hilbert space, and let $\mathcal{L}(\mathcal{H})$ denote the algebra of all bounded linear operators on $\mathcal{H}$. For commutative $n$ tuples of operators $A=\left(A_{1}, \ldots, A_{n}\right)$ and $B=\left(B_{1}, \ldots, B_{n}\right)$, let $R \equiv R_{A B}: \mathcal{L}(\not{H}) \rightarrow$ $\mathcal{L}(\not{H})$ denote the elementary operator defined by

$$
R(X)=A_{1} X B_{1}+\cdots+A_{n} X B_{n} \quad(X \in \mathcal{L}(\not)) .
$$

Elementary operators (in a more general Banach algebra context) were introduced by G. Lumer and M. Rosenblum [22], who computed the spectra of such operators in cases where the $A_{i}$ 's or $B_{i}$ 's are functionally related. Let $\sigma_{\mathrm{H}}(T)$ and $\sigma_{\mathrm{He}}(T)$ denote, respectively, the spectrum and Fredholm essential spectrum of an $n$-tuple $T \in \mathcal{L}(\mathcal{H})^{(n)}$ in the sense of R. Harte $[18,19,8]$. In Theorem 2.1 we derive the following expression for the essential spectrum of an elementary operator:

$$
\sigma_{\mathrm{e}}\left(R_{A B}\right)=\sigma_{\mathrm{H}}(A) \circ \sigma_{\mathrm{He}}(B) \cup \sigma_{\mathrm{He}}(A) \circ \sigma_{\mathrm{H}}(B) .
$$

(For subsets $\sigma, \tau \subset \mathbf{C}^{n}, \sigma \circ \tau=\left\{\alpha \circ \beta \equiv \sum \alpha_{i} \beta_{i}: \alpha=\left(\alpha_{1}, \ldots, \alpha_{n}\right) \in \sigma, \beta=\right.$ $\left.\left(\beta_{1}, \ldots, \beta_{n}\right) \in \tau\right\}$.)

As described below, this result supplements the description of $\sigma_{\mathrm{e}}\left(R_{A B}\right)$ obtained by $\mathrm{A}$. Carrillo and C. Hernández $[\mathbf{3}]$, and it closely parallels the following expression for the spectrum of $R_{A B}$ that we derive in the sequel from results of $\mathrm{R}$. Harte [19] and R. Curto [5]:

$$
\sigma\left(R_{A B}\right)=\sigma_{\mathrm{H}}(A) \circ \sigma_{\mathrm{H}}(B) .
$$

A well-known and useful consequence of Voiculescu's Theorem [27], due to C. Apostol [2, Lemma 2.2], is that if $T \in \mathcal{L}(\mathcal{H})$ and $\lambda \in \sigma_{\text {le }}(T)$ (the left essential spectrum of $T)$, then $U(T)^{-}$, the norm closure of the unitary orbit of $T$, contains an operator $S$ such that $\operatorname{dim} \operatorname{ker}(S-\lambda)=\infty$. In the course of proving Theorem 2.1, we obtain the following close analogue of this result for elementary operators: If $\lambda \in \sigma_{\mathrm{le}}\left(R_{A B}\right)$, then there exists an elementary operator $R_{A^{\prime} B^{\prime}} \in \mathcal{U}\left(R_{A B}\right)^{-}$such that $\operatorname{dim} \operatorname{ker}\left(R_{A^{\prime} B^{\prime}}-\lambda\right)=\infty$ (Corollary 2.9); if $\lambda \in \sigma_{\text {re }}\left(R_{A B}\right)$ (the right essential

Received by the editors April 6, 1984 and, in revised form, August 25, 1984.

1980 Mathematics Subject Classification. Primary 47A10, 47A53.

${ }^{1}$ Research supported by a National Science Foundation Research Grant. 
spectrum of $\left.R_{A B}\right)$, then there exists an elementary operator $R_{A^{\prime} B^{\prime}} \in \mathcal{U}\left(R_{A B}\right)^{-}$ such that $\operatorname{dim}\left(\mathcal{L}(\not{H}) / \operatorname{Ran}\left(R_{A^{\prime} B^{\prime}}-\lambda\right)^{-}\right)=\infty$ (Corollary 2.11).

We prove all of the preceding results, not just for elementary operators, but also for operators of the form $R_{A B} \mid J: J \rightarrow J$, where $(J,|| \cdot|| \mid)$ is an arbitrary norm ideal of $\mathcal{L}(\not{H})$. This extension is useful in obtaining applications to sums of tensor products of operators (as discussed in [10]).

For $A, B \in \mathcal{L}(\not{K})$, define $\tau_{A B}(X)=A X-X B$ and $S_{A B}(X)=A X B(X \in \mathcal{L}(\not{H}))$. For $R=\tau$ or $R=S$, and for $\lambda \in \rho_{\mathrm{SF}}(R)$ (the semi-Fredholm domain of $R$ ), we may compute index $(R-\lambda)$ in terms of spectral and algebraic invariants of $A$ and $B$ [9, 10, 11]. The problem of extending these results to arbitrary elementary operators remains open, even for $n=2$, and leads naturally to the problem of describing the isolated points of the Harte joint spectrum. In $\S 3$ we study this problem for a commuting pair of operators $(A, B)$, and we obtain some information concerning the location of isolated points (Proposition 3.4). We show that, in general, isolated points of the Harte-Fredholm domain of $(A, B)$ cannot be excised by a Riesz-type spectral decomposition (Example 3.5).

In the remainder of this section we recall the terminology and results that we require in the sequel. Let $\mathcal{X}$ denote a complex Banach space, and let $\mathcal{L}(\mathcal{X})$ denote the algebra of all bounded linear operators on $\chi$. For $A \in \mathcal{L}(\mathcal{X})$, let $\sigma(A)$ denote the spectrum of $A$, i.e.,

$$
\sigma(A)=\{\lambda \in \mathbf{C}: A-\lambda 1 x \text { is not 1-1 or not onto }\} .
$$

For a commutative $n$-tuple $A=\left(A_{1}, \ldots, A_{n}\right) \in \mathcal{L}(\mathcal{X})^{(n)}$, let $\sigma_{\mathrm{T}}(A)$ denote the Taylor spectrum of $A$ [25]. Let $\mathcal{K}(\mathcal{X})$ denote the closed 2-sided ideal of all compact operators in $\mathcal{L}(\mathcal{X})$, and let $A(\mathcal{X})=\mathcal{L}(\mathcal{X}) / \mathcal{K}(\mathcal{X})$. For $A \in \mathcal{L}(\mathcal{X})$, let $\tilde{A}$ denote the image of $A$ in $A(X)$ under the canonical projection, and let $\sigma_{\mathrm{e}}(a)=\sigma(\tilde{A})$ denote the Fredholm essential spectrum of $A$. For an $n$-tuple $A, \sigma_{\mathrm{Te}}(A)$ denotes the Taylor essential spectrum of $A[\mathbf{4}]$.

Let $A$ denote a complex Banach algebra with identity 1 . For $A=\left(A_{1}, \ldots, A_{n}\right) \in$ $A^{(n)}$, the left spectrum of $A$ (relative to $A$ ) is defined by

$$
\begin{aligned}
\sigma_{1}(A)=\left\{\lambda \in \mathbf{C}^{n}:\right. & \text { There is no solution } X \in A^{(n)} \\
& \text { to the equation } \left.X_{1}\left(A_{1}-\lambda_{1}\right)+\cdots+X_{n}\left(A_{n}-\lambda_{n}\right)=1\right\} .
\end{aligned}
$$

The right spectrum of $A, \sigma_{\mathrm{r}}(A)$, is defined analogously, and the Harte spectrum of $A$ is given by $\sigma_{\mathrm{H}}(A)=\sigma_{\mathrm{l}}(A) \cup \sigma_{\mathrm{r}}(A)[\mathbf{1 8}, \mathbf{1 9}]$.

For a Banach space $X, \mathcal{A}=\mathcal{A}(\mathcal{X})$, and $A \in \mathcal{L}(\mathcal{X})^{(n)}$, let $\tilde{A}=\left(\tilde{A}_{1}, \ldots, \tilde{A}_{n}\right) \in$ $A(X)^{(n)}$, and let $\sigma_{\mathrm{le}}(A)=\sigma_{\mathrm{l}}(\tilde{A})$, the left essential spectrum of $\mathcal{A}$, and $\sigma_{\mathrm{re}}(A)=$ $\sigma_{\mathrm{r}}(\tilde{A})$, the right essential spectrum of $A$. The Harte essential spectrum of $A$ is given by $\sigma_{\mathrm{He}}(A)=\sigma_{\mathrm{le}}(A) \cup \sigma_{\mathrm{re}}(A)$. If $A \in \mathcal{L}(\mathcal{X})$, then $\sigma(A)=\sigma_{\mathrm{H}}(A)=\sigma_{\mathrm{T}}(A)$ and $\sigma_{\mathrm{e}}(A)=\sigma_{\mathrm{He}}(A)=\sigma_{\mathrm{Te}}(A)$; for $n>1$ and $A \in \mathcal{L}(\not{H})^{(n)}, \sigma_{\mathrm{H}}(A) \subset \sigma_{\mathrm{T}}(A)$ and $\sigma_{\mathrm{He}}(A) \subset \sigma_{\mathrm{Te}}(A)($ see $[\mathbf{2 5}])$.

In $[19$, Theorem 3.5] R. Harte obtained the following identities for the left and right spectra of elementary operators:

$$
\sigma_{\mathrm{l}}\left(R_{A B}\right)=\sigma_{\mathrm{l}}(A) \circ \sigma_{\mathrm{r}}(B)
$$

and

$$
\sigma_{\mathrm{r}}\left(R_{A B}\right)=\sigma_{\mathrm{r}}(A) \circ \sigma_{\mathrm{l}}(B)
$$


thus,

$$
\sigma\left(R_{A B}\right)=\sigma_{\mathrm{l}}(A) \circ \sigma_{\mathrm{r}}(B) \cup \sigma_{\mathrm{r}}(A) \circ \sigma_{\mathrm{l}}(B) .
$$

With the aid of (1.3) and (1.4), R. Curto [5] derived the following expression for $\sigma\left(R_{A B}\right)$ in terms of Taylor spectra:

$$
\sigma\left(R_{A B}\right)=\sigma_{\mathrm{T}}(A) \circ \sigma_{\mathrm{T}}(B) .
$$

Thus,

$$
\begin{aligned}
\sigma_{\mathrm{H}}(A) \circ \sigma_{\mathrm{H}}(B) \subset \sigma_{\mathrm{T}}(A) \circ \sigma_{\mathrm{T}}(B)=\sigma\left(R_{A B}\right) \\
\quad=\sigma_{\mathrm{l}}(A) \circ \sigma_{\mathrm{r}}(B) \cup \sigma_{r}(A) \circ \sigma_{1}(B) \subset \sigma_{\mathrm{H}}(A) \circ \sigma_{\mathrm{H}}(B),
\end{aligned}
$$

whence we deduce (1.2) and also the identity

$$
\sigma_{\mathrm{H}}(A) \circ \sigma_{\mathrm{H}}(B)=\sigma_{\mathrm{T}}(A) \circ \sigma_{\mathrm{T}}(B) .
$$

Formula (1.7) is of interest because there is at present no concrete prescription for computing $\sigma_{\mathrm{T}}(A) \backslash \sigma_{\mathrm{H}}(A)$ (even for pairs of operators, $n=2$ ), and (1.7) may prove useful in such computations. (The results of $\S 3$ may also be useful in this regard.) It would be interesting to discover a proof of (1.7) that is independent of results about elementary operators; we know of no such argument at present.

Identity (1.1) is the analogue of (1.2) for essential spectra. In [7] R. Curto obtains the following analogue of (1.7) for essential spectra:

$$
\begin{aligned}
\sigma_{\mathrm{Te}}(A) \circ \sigma_{\mathrm{T}}(B) \cup \sigma_{\mathrm{T}}(A) \circ \sigma_{\mathrm{Te}}(B) \\
=\sigma_{\mathrm{He}}(A) \circ \sigma_{\mathrm{H}}(B) \cup \sigma_{\mathrm{H}}(A) \circ \sigma_{\mathrm{He}}(B),
\end{aligned}
$$

from which follows (via (1.1)) the following analogue of (1.6):

$$
\sigma_{\mathrm{e}}\left(R_{A B}\right)=\sigma_{\mathrm{Te}}(A) \circ \sigma_{\mathrm{T}}(B) \cup \sigma_{\mathrm{T}}(A) \circ \sigma_{\mathrm{Te}}(B) .
$$

Results such as (1.1) (and hence (1.9)) depend on analogues of (1.3)-(1.5) for essential spectra. These are the results of A. Carrillo and C. Hernández that we next describe.

For $n$-tuples of Hilbert space operators $A$ and $B$, let

$$
\begin{aligned}
& \sigma_{\mathrm{lr}} \equiv \sigma_{\mathrm{lr}}(A, B)=\sigma_{\mathrm{l}}(A) \circ \sigma_{\mathrm{re}}(B) \cup \sigma_{\mathrm{le}}(A) \circ \sigma_{\mathrm{r}}(B), \\
& \sigma_{\mathrm{rl}} \equiv \sigma_{\mathrm{rl}}(A, B)=\sigma_{\mathrm{r}}(A) \circ \sigma_{\mathrm{le}}(B) \cup \sigma_{\mathrm{re}}(A) \circ \sigma_{\mathrm{l}}(B), \\
& \sigma_{\mathrm{ll}} \equiv \sigma_{\mathrm{ll}}(A, B)=\sigma_{\mathrm{l}}(A) \circ \sigma_{\mathrm{le}}(B) \cup \sigma_{\mathrm{le}}(A) \circ \sigma_{\mathrm{l}}(B), \quad \text { and }, \\
& \sigma_{\mathrm{rr}} \equiv \sigma_{\mathrm{rr}}(A, B)=\sigma_{\mathrm{r}}(A) \circ \sigma_{\mathrm{re}}(B) \cup \sigma_{\mathrm{re}}(A) \circ \sigma_{\mathrm{r}}(B) .
\end{aligned}
$$

In [3, Corollary 1] A. Carrillo and C. Hernández proved the following analogues of (1.3)-(1.5):

$$
\begin{gathered}
\sigma_{\mathrm{le}}\left(R_{A B}\right)=\sigma_{\mathrm{lr}}(A, B), \\
\sigma_{\mathrm{re}}\left(R_{A B}\right)=\sigma_{\mathrm{rl}}(A, B), \\
\sigma_{\mathrm{e}}\left(R_{A B}\right)=\sigma_{\mathrm{lr}}(A, B) \cup \sigma_{\mathrm{rl}}(A, B) .
\end{gathered}
$$

In $\S 2$ our proofs of the inclusions

$$
\sigma_{\mathrm{lr}}(A, B) \subset \sigma_{\mathrm{le}}\left(R_{A B}\right) \quad \text { and } \quad \sigma_{\mathrm{rl}}(A, B) \subset \sigma_{\mathrm{re}}\left(R_{A B}\right)
$$


yield the stronger conclusions of Corollaries 2.9 and 2.11 (described above). (Propositions 2.8 and 2.10 were found independently of $[\mathbf{3}]$ and were announced in [12].)

Clearly, one may extend the definition of an elementary operator to the case where the coefficient sequences are not necessarily commutative. We consider only the commutative case in the sequel, but we note that several of the results of $\S 2$ (e.g., Lemmas 2.2-2.7, Propositions 2.8 and 2.10) can be extended to the general case with no change in the proofs. On the other hand, Theorem 2.1 does not extend to the noncommutative setting.

The author wishes to thank Professsors A. Carrillo and C. Hernández for early receipt of $[3]$. The author is also grateful to Professor R. Curto for several interesting conversations concerning the topics discussed in this paper.

2. Essential spectra of the operators $R_{J}$. In this section we prove (1.1). In the sequel, $A=\left(A_{1}, \ldots, A_{n}\right)$ and $B=\left(B_{1}, \ldots, B_{n}\right)$ denote commutative $n$-tuples of operators in $\mathcal{L}(\mathscr{H})$. Let $(J,|||\cdot|||)$ denote a symmetric norm ideal of $\mathcal{L}(\mathscr{H})$ in the sense of $[15,24]$; as a notational convenience we extend this notation to include $J=\mathcal{L}(\mathscr{H})$. Since $J$ is a 2 -sided ideal of $\mathcal{L}(\not{H}), J$ is invariant under $R_{A B}$, and the restriction of $R_{A B}$ to $J$, which we denote by $R_{J}$, defines a bounded operator on the Banach space $J$.

Let $x, y \in \not{H}$ and let $x \otimes y \in \mathcal{L}(\not{H})$ denote the rank-one operator defined by $(x \otimes y)(h)=(h, y) x(h \in \mathcal{H})$. Since $J$ contains each finite rank operator, it is clear that $S_{x \otimes x, y \otimes y} \mid J \neq 0$ whenever $x \neq 0$ and $y \neq 0$. If $L, K \in \mathcal{K}(\mathcal{H})$, then $S_{L K} \mid J$ is a compact operator in $\mathcal{L}(J)$. (Indeed, $S_{L K} \mid J$ may be norm-approximated in $\mathcal{L}(J)$ by finite rank operators of the form $S_{F G} \mid J$, where $F$ and $G$ are finite rank operators.) Using these observations, it follows from [3, Theorem 2] (applied to the Banach space $\mathcal{Y}=\mathcal{L}(J))$ that $(1.8)-(1.10)$ admit exact analogues for the operators $R_{J}$ :

$$
\begin{aligned}
\sigma_{\mathrm{le}}\left(R_{J}\right) & =\sigma_{\mathrm{lr}}(A, B) ; \\
\sigma_{\mathrm{re}}\left(R_{J}\right) & =\sigma_{\mathrm{rl}}(A, B) ; \\
\sigma_{\mathrm{e}}\left(R_{J}\right) & =\sigma_{\mathrm{lr}}(A, B) \cup \sigma_{\mathrm{rl}}(A, B) .
\end{aligned}
$$

Our goal is to prove the following alternate expression for the essential spectrum of $R J$.

THEOREM 2.1. $\sigma_{\mathrm{e}}\left(R_{J}\right)=\sigma_{\mathrm{H}}(A) \circ \sigma_{\mathrm{He}}(B) \cup \sigma_{\mathrm{He}}(A) \circ \sigma_{\mathrm{H}}(B)$.

Before beginning the proof we require some additional notation. For a Banach space $\mathcal{X}$ and $T \in \mathcal{L}(\mathcal{X})$, we set

$$
\mathcal{U}(T)=\left\{U^{-1} T U: U: \mathcal{X} \rightarrow \mathcal{X} \text { is an isometric isomorphism }\right\} .
$$

Let $\operatorname{ker} T$ and $\operatorname{Ran} T$ denote, respectively, the kernel and range of $T$. Let

$$
\rho_{\mathrm{SF}}(T)=\{\lambda \in \mathbf{C}: T-\lambda \text { is a semi-Fredholm operator }\},
$$

the semi-Fredholm domain of $T[\mathbf{2 1}]$. For $\lambda \in \mathbf{C}$, let $\operatorname{nul}(T-\lambda)=\operatorname{dim} \operatorname{ker} T-$ $\lambda, \operatorname{def}(T-\lambda)=\operatorname{dim}\left(\mathcal{X} / \operatorname{Ran}(T-\lambda)^{-}\right)$, and for $\lambda \in \rho_{\mathrm{SF}}(T)$, let $\operatorname{ind}(T-\lambda)=$ nul $(T-\lambda)-\operatorname{def}(T-\lambda)$ (the index of $T-\lambda)[\mathbf{2 1}]$. Note that $\{\lambda \in \mathbf{C}: \operatorname{nul}(T-\lambda)=$ $\infty\} \subset \sigma_{\text {le }}(T)$ and $\{\lambda \in \mathbf{C}: \operatorname{def}(T-\lambda)=\infty\} \subset \sigma_{\text {re }}(T)$ [28, Theorem 4].

Let $A$ and $A^{\prime}$ be $n$-tuples of operators; $A$ and $A^{\prime}$ are approximately unitarily equivalent, $A \approx_{\mathrm{a}} A^{\prime}$, if there exists a sequence of unitary operators $\left\{U_{k}\right\}_{k=1}^{\infty} \subset \mathcal{L}(\not{K})$ such that $\lim \left\|A_{i}^{\prime}-U_{k}^{*} A_{i} U_{k}\right\|=0(1 \leq i \leq n)[\mathbf{2 7}] . \quad A$ and $A^{\prime}$ are approximately 
similar, $A \sim_{\mathrm{a}} A^{\prime}$, if there exists a sequence of invertible operators $\left\{X_{k}\right\}_{k=1}^{\infty} \subset \mathcal{L}(\not{H})$ such that $\sup _{k}\left\|X_{k}\right\|<\infty, \sup _{k}\left\|X_{k}^{-1}\right\|<\infty$, and $\lim \left\|A_{i}^{\prime}-X_{k}^{-1} A_{i} X_{k}\right\|=0(1 \leq$ $i \leq n)[\mathbf{1 6}]$.

LEMMA 2.2. If $A \sim_{\mathrm{a}} A^{\prime}$ and $B \sim_{\mathrm{a}} B^{\prime}$, then, for $\lambda \in \mathbf{C}, R_{J}(A, B)-\lambda$ is semiFredholm if and only if $R_{J}\left(A^{\prime}, B^{\prime}\right)-\lambda$ is semi-Fredholm, and, in this case,

$$
\operatorname{ind}\left(R_{J}(A, B)-\lambda\right)=\operatorname{ind}\left(R_{J}\left(A^{\prime}, B^{\prime}\right)-\lambda\right) ;
$$

moreover,

$$
\sigma_{\mathrm{le}}\left(R_{J}(A, B)\right)=\sigma_{\mathrm{le}}\left(R_{J}\left(A^{\prime}, B^{\prime}\right)\right) \quad \text { and } \quad \sigma_{\mathrm{re}}\left(R_{J}(A, B)\right)=\sigma_{\mathrm{re}}\left(R_{J}\left(A^{\prime}, B^{\prime}\right)\right) .
$$

If $A \approx_{\mathrm{a}} A^{\prime}$ and $B \approx_{\mathrm{a}} B^{\prime}$, then $R_{\mathcal{J}}\left(A^{\prime}, B^{\prime}\right) \in U\left(R_{J}(A, B)\right)^{-}$.

PrOOF. The first part follows by a direct adaptation of the proof of [11, Proposition 2.5] concerning the operator $S_{J}(A, B)$. For the second part, let $\left\{U_{k}\right\}$ and $\left\{V_{k}\right\}$ denote sequences of unitary operators on $\mathcal{H}$ such that $\left\|A_{i}^{\prime}-U_{k}^{*} A_{i} U_{k}\right\| \rightarrow 0$ and $\left\|B_{i}^{\prime}-V_{k}^{*} B_{i} V_{k}\right\| \rightarrow 0(1 \leq i \leq n)$. For $T \in \mathcal{L}(\mathcal{H})$, let $L_{T}$ and $R_{T}$ denote the operators on $J$ defined by $L_{T}(X)=T X$ and $R_{T}(X)=X T$. Clearly, $W_{k}=L_{U_{k}} R_{V_{k}^{*}}$ is an isometric isomorphism of $J$ and

$$
\left\|W_{k}^{-1} R_{J}(A, B) W_{k}-R_{J}\left(A^{\prime}, B^{\prime}\right)\right\| \rightarrow 0 .
$$

For ease of reference we next recall two known results concerning left and, respectively, left essential spectra.

LEMMA 2.3 [8, LEMMA 2.4]. For $A \in \mathcal{L}(\mathcal{H})^{(n)}$ and $\lambda \in \mathbf{C}^{n}$ the following are equivalent:

(i) $\lambda \in \sigma_{\mathrm{l}}(A)$;

(ii) $\sum\left(A_{i}-\lambda_{i}\right)^{*}\left(A_{i}-\lambda_{i}\right)$ is not invertible.

LeMma 2.4 [8, TheOREM 2.6; 14]. For $A \in \mathcal{L}(\mathcal{H})^{(n)}$ and $\lambda \in \mathbf{C}^{(n)}$ the following are equivalent:

(i) $\lambda \in \sigma_{\text {le }}(A)$, i.e., there is no solution $X \in \mathcal{L}(\mathcal{H})^{(n)}$ to $\sum \tilde{X}_{i}\left(\tilde{A}_{i}-\lambda_{i}\right)=1$;

(ii) there exists an infinite rank orthogonal projection $P \in \mathcal{L}(\mathcal{H})$ such that $\left(A_{i}-\lambda_{i}\right) P \in \mathcal{K}(\mathcal{K}), 1 \leq i \leq n$;

(iii) there exists an orthonormal sequence $\left\{e_{k}\right\}_{k=1}^{\infty}$ such that $\lim _{k}\left\|\left(A_{i}-\lambda_{i}\right) e_{k}\right\|=$ $0,1 \leq i \leq n$

(iv) $\sum\left(A_{i}-\lambda_{i}\right)^{*}\left(A_{i}-\lambda_{i}\right)$ is not Fredholm.

The following result is a direct adaptation of the aforementioned result of $\mathrm{C}$. Apostol [2, Lemma 2.2], so we omit the proof.

LEMMA 2.5. If $A \in \mathcal{L}(\not{H})^{(n)}$ and $\lambda \in \sigma_{\mathrm{le}}(A)$, then there exist $A^{\prime} \approx_{\mathrm{a}} A$ and an infinite rank projection $P \in \mathcal{L}(\mathcal{H})$ such that $\left(A_{i}^{\prime}-\lambda_{i}\right) P=0,1 \leq i \leq n$.

For $A \in \mathcal{L}(\mathcal{H})^{(n)}$, let $\sigma_{\mathrm{p}}(A)$ denote the joint point spectrum of $A_{1}, \ldots, A_{n}$, i.e.,

$$
\begin{aligned}
\sigma_{\mathrm{p}}(A)=\left\{\lambda \in \mathbf{C}^{n}:\right. & \text { There exists } x \in \mathcal{H}, x \neq 0, \\
& \text { such that } \left.\left(A_{i}-\lambda_{i}\right) x=0,1 \leq i \leq n\right\} .
\end{aligned}
$$

LEMMA 2.6. If $\lambda \in \sigma_{1}(A)$, then there exists $A^{\prime} \approx_{\mathrm{a}} A$ such that $\lambda \in \sigma_{\mathrm{p}}\left(A^{\prime}\right)$.

ProOF. This is the analogue of [11, Lemma 2.12] for $n>1$. If $\lambda \in \sigma_{\text {le }}(A)$, Lemma 2.5 implies that there exists $A^{\prime} \approx{ }_{\mathrm{a}} A$ and an infinite rank projection $P \in$ 
$\mathcal{L}(\not)$ such that $\left(A_{i}-\lambda_{i}\right) P=0(1 \leq i \leq n)$. Clearly, $P \nvdash \subset \bigcap_{i=1}^{n} \operatorname{ker}\left(A_{i}^{\prime}-\lambda_{i}\right)$, so $\lambda \in \sigma_{\mathrm{p}}\left(A^{\prime}\right)$. Suppose $\lambda \in \sigma_{\mathrm{l}}(A) \backslash \sigma_{\mathrm{le}}(A)$. Lemmas 2.3 and 2.4 imply that the positive operator $M=\sum\left(A_{i}-\lambda_{i}\right)^{*}\left(A_{i}-\lambda_{i}\right)$ is Fredholm but not invertible, and thus has nontrivial kernel $\mathcal{M}$. If $x$ is a nonzero vector in $\mathcal{M}$, then $\sum\left\|\left(A_{i}-\lambda_{i}\right) x\right\|^{2}=$ $(M x, x)=0$, so $x \in \operatorname{ker}\left(A_{i}-\lambda_{i}\right)(1 \leq i \leq n)$ and, thus, $\lambda \in \sigma_{\mathrm{p}}(A)$.

By considering adjoints we also obtain

LEMMA 2.7. Let $A \in \mathcal{L}(\mathcal{H})^{(n)}$.

(i) If $\lambda \in \sigma_{\mathrm{re}}(A)$, then there exist $A^{\prime} \approx_{\mathrm{a}} A$ and an infinite rank projection $P$ such that $\left(A_{i}^{\prime}-\lambda_{i}\right)^{*} P=0(1 \leq i \leq n)$.

(ii) If $\lambda \in \sigma_{\mathrm{r}}(A)$, either $\lambda \in \sigma_{\mathrm{re}}(A)$ or $\bar{\lambda} \in \sigma_{\mathrm{p}}\left(A^{*}\right)$ (where $\bar{\lambda}=\left(\bar{\lambda}_{1}, \ldots, \bar{\lambda}_{n}\right)$ and $\left.A^{*}=\left(A_{1}^{*}, \ldots, A_{n}^{*}\right)\right)$.

(iii) If $\lambda \in \sigma_{\mathrm{r}}(A)$, then there exists $A^{\prime} \approx_{\mathrm{a}} A$ such that $\bar{\lambda} \in \sigma_{\mathrm{p}}\left(A^{\prime *}\right)$.

Proposition 2.8. If $\lambda \in \sigma_{\mathrm{lr}}(A, B)$, then there exists

$$
R_{J}\left(A^{\prime}, B^{\prime}\right) \in \mathcal{U}\left(R_{J}(A, B)\right)^{-}
$$

such that $\operatorname{nul}\left(R_{J}\left(A^{\prime}, B^{\prime}\right)-\lambda\right)=\infty$; in particular, $\sigma_{\mathrm{lr}}(A, B) \subset \sigma_{\mathrm{le}}\left(R_{J}(A, B)\right)$, and if $R_{J}(A, B)-\lambda$ is semi-Fredholm, then $\operatorname{ind}\left(R_{J}(A, B)-\lambda\right)=+\infty$.

Proof. Let $\lambda \in \sigma_{\mathrm{lr}}(A, B)$ and suppose $\lambda=\alpha \circ \beta$ with $\alpha \in \sigma_{\mathrm{le}}(A), \beta \in$ $\sigma_{\mathrm{r}}(B)$ or $\alpha \in \sigma_{\mathrm{l}}(A), \beta \in \sigma_{\mathrm{re}}(B)$. It follows from Lemmas 2.5-2.7 that there exist $A^{\prime} \approx_{\mathrm{a}} A, B^{\prime} \approx_{\mathrm{a}} B$, and nonzero orthogonal projections $P$ and $Q$ such that

(i) $\left(A_{i}^{\prime}-\alpha_{i}\right) P=0(1 \leq i \leq n)$,

(ii) $\left(B_{i}^{\prime}-\beta_{i}\right)^{*} Q=0(1 \leq i \leq n)$,

(iii) $P$ or $Q$ has infinite rank.

Let $H_{1}$ and $H_{2}$ denote copies of $\mathscr{H}$, and consider the decompositions $\mathscr{H}_{1}=P \mathscr{H}_{1} \oplus$ $(1-P) \mathscr{H}_{1}$ and $\mathscr{H}_{2}=Q \mathscr{H}_{2} \oplus(1-Q) \mathscr{H}_{2}$. Relative to these decompositions, the matrices of $A_{i}^{\prime} \in \mathcal{L}\left(\mathscr{H}_{1}\right), B_{i}^{\prime} \in \mathcal{L}\left(\mathscr{H}_{2}\right)(1 \leq i \leq n)$, and $X \in \mathcal{L}(\mathscr{H})=\mathcal{L}\left(\mathscr{H}_{2}, \not{H} \mathcal{H}_{1}\right)$ are of the form

$$
A_{i}^{\prime}=\left(\begin{array}{cc}
\alpha_{i} & A_{12}^{(i)} \\
0 & A_{22}^{(i)}
\end{array}\right), \quad B_{i}^{\prime}=\left(\begin{array}{cc}
\beta_{i} & 0 \\
B_{21}^{(i)} & B_{22}^{(i)}
\end{array}\right),
$$

and

$$
X=\left(\begin{array}{ll}
X_{11} & X_{12} \\
X_{21} & X_{22}
\end{array}\right) .
$$

Let $\mathcal{F}=\left\{X \in \mathcal{L}(\mathcal{H}): X_{12}, X_{21}, X_{22}\right.$ are zero operators and $X_{11}: Q \mathscr{H}_{2} \rightarrow P \mathscr{H}_{1}$ is a finite rank operator $\}$. A calculation shows that for $X \in \mathcal{F}, A_{i}^{\prime} X B_{i}^{\prime}-\alpha_{i} \beta_{i} X=0$, so $\left(R\left(A^{\prime}, B^{\prime}\right)-\lambda\right)(X)=0$. Since $P$ or $Q$ has infinite rank, $\mathcal{F}$ is infinite dimensional; moreover, $\exists \subset J$. Thus

$$
\operatorname{nul}\left(R_{J}\left(A^{\prime}, B^{\prime}\right)-\lambda\right) \geq \operatorname{dim} \xi=\infty .
$$

It follows that $\lambda \in \sigma_{\text {le }}\left(R_{J}\left(A^{\prime}, B^{\prime}\right)\right)[\mathbf{2 8}]$ and, if $R_{J}\left(A^{\prime}, B^{\prime}\right)-\lambda$ is semi-Fredholm, that $\operatorname{ind}\left(R_{J}\left(A^{\prime}, B^{\prime}\right)-\lambda\right)=+\infty$. The result now follows from Lemma 2.2.

Corollary 2.9. If $\lambda \in \sigma_{\mathrm{le}}\left(R_{J}(A, B)\right)$, then there exists

$$
R_{J}\left(A^{\prime}, B^{\prime}\right) \in \mathcal{U}\left(R_{J}(A, B)\right)^{-}
$$

such that $\operatorname{nul}\left(R_{\mathcal{J}}\left(A^{\prime}, B^{\prime}\right)-\lambda\right)=\infty$.

PROOF. The result follows from Proposition 2.8 and (2.1). 
Proposition 2.10. If $\lambda \in \sigma_{\mathrm{rl}}(A, B)$, then there exists

$$
R_{J}\left(A^{\prime}, B^{\prime}\right) \in \mathcal{U}\left(R_{J}(A, B)\right)^{-}
$$

such that $\operatorname{def}\left(R_{J}\left(A^{\prime}, B^{\prime}\right)-\lambda\right)=\infty ;$ thus

$$
\sigma_{\mathrm{rl}}(A, B) \subset \sigma_{\mathrm{re}}\left(R_{J}(A, B)\right),
$$

and if $R_{J}(A, B)-\lambda$ is semi-Fredholm, then $\operatorname{ind}\left(R_{J}(A, B)-\lambda\right)=-\infty$.

PROOF. Let $\lambda \in \sigma_{\mathrm{rl}}(A, B)$ and suppose $\lambda=\alpha \circ \beta$, with $\alpha \in \sigma_{\mathrm{re}}(A), \beta \in \sigma_{\mathrm{l}}(B)$ or $\alpha \in \sigma_{\mathrm{r}}(A), \beta \in \sigma_{\mathrm{le}}(B)$. Lemmas 2.5-2.7 imply that there exist $A^{\prime} \approx_{\mathrm{a}} A, B^{\prime} \approx_{\mathrm{a}} B$, and nonzero orthogonal projections $P$ and $Q$ such that

(i) $\left(A_{i}^{\prime}-\alpha_{i}\right)^{*} P=0(1 \leq i \leq n)$,

(ii) $\left(B_{i}^{\prime}-\beta_{i}\right) Q=0(1 \leq i \leq n)$,

(iii) $P$ or $Q$ has infinite rank.

Let $H_{1}$ and $H_{2}$ denote copies of $\mathscr{H}$; thus $H_{1}=P K_{1} \oplus(1-P) H_{1}$ and $H_{2}=$ $Q H_{2} \oplus(1-Q) H_{2}$. Relative to these decompositions, a calculation shows that, for $X \in J, Y \equiv\left(R_{J}\left(A^{\prime}, B^{\prime}\right)-\lambda\right)(X)$ satisfies $P Y Q=0$, whence $\operatorname{def}\left(R_{J}\left(A^{\prime}, B^{\prime}\right)-\lambda\right)=$ $\infty$, and the result follows from [28] and Lemma 2.2 (as in the proof of Proposition 2.8).

COROLlaRY 2.11. If $\lambda \in \sigma_{\mathrm{re}}\left(R_{J}(A, B)\right)$, then there exists

$$
R_{J}\left(A^{\prime}, B^{\prime}\right) \in \mathcal{U}\left(R_{J}(A, B)\right)^{-}
$$

such that $\operatorname{def}\left(R_{J}\left(A^{\prime}, B^{\prime}\right)\right)=\infty$.

PROOF. The result follows from Proposition 2.10 and (2.2).

Proposition 2.12. $\sigma_{\mathrm{ll}}(A, B) \subset \sigma_{\mathrm{e}}\left(R_{\jmath}(A, B)\right)$.

ProOF. The proof is an extension of the argument given in [9, Theorem 3.1]. Let $\lambda \in \sigma_{\mathrm{ll}}(A, B)$ and suppose $\lambda=\alpha \circ \beta$, with $\alpha \in \sigma_{\mathrm{le}}(A), \beta \in \sigma_{\mathrm{l}}(B)$ or $\alpha \in$ $\sigma_{\mathrm{l}}(A), \beta \in \sigma_{\mathrm{le}}(B)$. Lemmas 2.3 and 2.4 imply that there exist $A^{\prime} \approx_{\mathrm{a}} A, B^{\prime} \approx_{\mathrm{a}} B$, and nonzero orthogonal projections $P$ and $Q$ such that

(i) $\left(A_{i}^{\prime}-\alpha_{i}\right) P=0(1 \leq i \leq n)$,

(ii) $\left(B_{i}^{\prime}-\beta_{i}\right) Q=0(1 \leq i \leq n)$,

(iii) $P$ or $Q$ is an infinite rank projection.

Note that $A^{\prime}$ and $B^{\prime}$ are commutative $n$-tuples.

Let $H_{1}$ and $H_{2}$ denote copies of $H_{;}$; thus $H_{1}=P H_{1} \oplus(1-P) H_{1}$ and $H_{2}=$ $Q \mathscr{H}_{2} \oplus(1-Q) \mathscr{H}_{2}$. Relative to these decompositions, the operator matrices of $A_{i}^{\prime} \in$

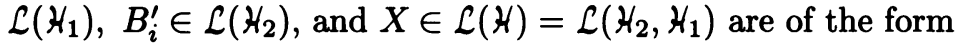

$$
A_{i}^{\prime}=\left(\begin{array}{cc}
\alpha_{i} & A_{12}^{(i)} \\
0 & A_{22}^{(i)}
\end{array}\right), \quad B_{i}^{\prime}=\left(\begin{array}{cc}
\beta_{i} & B_{12}^{(i)} \\
0 & B_{22}^{(i)}
\end{array}\right), \quad X=\left(\begin{array}{cc}
X_{11} & X_{12} \\
X_{21} & X_{22}
\end{array}\right) .
$$

For $V \in \mathcal{L}\left(Q \mathscr{H}_{2}, P \mathscr{H}_{1}\right)$, let

$$
M(V)=\left(\begin{array}{ll}
V & 0 \\
0 & 0
\end{array}\right) \in \mathcal{L}\left(\mathscr{H}_{2}, \mathscr{H}_{1}\right) .
$$

Let $\mathcal{M}=\left\{M(V): V \in \mathcal{L}\left(Q \mathscr{H}_{2}, P \mathscr{H}_{1}\right)\right.$ and $V$ is a finite rank operator $\}$. Since $P$ or $Q$ has infinite rank, $\mathcal{M}$ is an infinite-dimensional linear subspace of $\mathcal{J} \subset \mathcal{L}(\mathcal{H})$. 
Let $R_{J}^{\prime}=R_{J}\left(A^{\prime}, B^{\prime}\right)$. From Lemma 2.2 , it suffices to show that $R_{J}^{\prime}-\lambda$ is not Fredholm. We first show that

$$
\operatorname{dim} \operatorname{ker}\left(R_{J}^{\prime}-\lambda\right) \geq \operatorname{dim}\left(\mathcal{M} \cap \operatorname{Ran}\left(R_{J}^{\prime}-\lambda\right)\right) .
$$

Let $M \in \mathcal{M} \cap \operatorname{Ran}\left(R_{J}^{\prime}-\lambda\right)$; thus $M=M(V)$ for some finite rank operator $V \in$ $\mathcal{L}\left(Q \mathscr{H}_{2}, P \mathscr{H}_{1}\right)$; to prove $(*)$, it suffices to show that $\operatorname{ker}\left(R_{J}^{\prime}-\lambda\right)$ contains an operator of the form

$$
\left(\begin{array}{ll}
V & * \\
0 & *
\end{array}\right) .
$$

Let $X \in J$ be an operator such that $\left(R_{J}^{\prime}-\lambda\right)(X)=M(V)$. Let $W(V)=$ $\sum\left(A_{j}^{\prime}-\alpha_{j}\right) X B_{j}^{\prime}$ and note that

$$
\begin{aligned}
W(V) & =\sum\left[\left(A_{j}^{\prime}-\alpha_{j}\right) X B_{j}^{\prime}+\alpha_{j} X\left(B_{j}^{\prime}-\beta_{j}\right)\right]-\sum \alpha_{j} X\left(B_{j}^{\prime}-\beta_{j}\right) \\
& =\left(R_{J}^{\prime}-\lambda\right)(X)-\sum \alpha_{j} X\left(B_{j}^{\prime}-\beta_{j}\right)=M(V)-\sum \alpha_{j} X\left(B_{j}^{\prime}-\beta_{j}\right) .
\end{aligned}
$$

For each $j$, the matrix of $X\left(B_{j}^{\prime}-\beta_{j}\right)$ is of the form $\left(\begin{array}{l}0 * \\ 0 *\end{array}\right)$, and it follows that the matrix of $W(V)$ is of the form $(* *)$. We claim that $W(V) \in \operatorname{ker}\left(R_{J}^{\prime}-\lambda\right)$; to show this, it suffices to verify that $\left(A_{j}^{\prime}-\alpha_{j}\right) X B_{j}^{\prime} \in \operatorname{ker}\left(R_{J}^{\prime}-\lambda\right)(1 \leq j \leq n)$. Now

$$
\begin{aligned}
\left(R_{J}^{\prime}-\right. & \lambda)\left(\left(A_{j}^{\prime}-\alpha_{j}\right) X B_{j}^{\prime}\right) \\
& =\sum_{i=1}^{n}\left[\left(A_{i}^{\prime}-\alpha_{i}\right)\left(\left(A_{j}^{\prime}-\alpha_{j}\right) X B_{j}^{\prime}\right) B_{i}^{\prime}+\left(\alpha_{i}\left(A_{j}^{\prime}-\alpha_{j}\right) X B_{j}^{\prime}\right)\left(B_{i}^{\prime}-\beta_{i}\right)\right] \\
& =\left(A_{j}^{\prime}-\alpha_{j}\right)\left(\sum_{i=1}^{n}\left[\left(A_{i}^{\prime}-\alpha_{i}\right) X B_{i}^{\prime}+\alpha_{i} X\left(B_{i}^{\prime}-\beta_{i}\right)\right]\right) B_{j}^{\prime} \\
& =\left(A_{j}^{\prime}-\alpha_{j}\right) M(V) B_{j}^{\prime}=0
\end{aligned}
$$

(since clearly $\left(A_{j}^{\prime}-\alpha_{j}\right) M(V)=0$ ).

Thus $\operatorname{dim} \operatorname{ker}\left(R_{J}^{\prime}-\lambda\right) \geq \operatorname{dim}\left(\mathcal{M} \cap \operatorname{Ran}\left(R_{J}^{\prime}-\lambda\right)\right)$. We may thus assume that $\operatorname{Ran}\left(R_{J}^{\prime}-\lambda\right)$ is closed and $n \equiv \operatorname{dim}\left(\mathcal{M} \cap \operatorname{Ran}\left(R_{J}^{\prime}-\lambda\right)\right)<\infty$, for otherwise $\lambda \in \sigma_{\mathbf{e}}\left(R_{J}^{\prime}\right)$. We claim that in this case $\operatorname{def}\left(R_{J}^{\prime}-\lambda\right)=\infty$. If $n>0$, let $\left\{V_{i}\right\}_{i=1}^{n} \subset \mathcal{L}\left(Q \mathscr{H}_{2}, P \mathscr{H}_{1}\right)$ be a sequence of finite rank operators such that $\left\{M\left(V_{i}\right)\right\}_{i=1}^{n}$ is a basis for $\mathcal{M} \cap \operatorname{Ran}\left(R_{J}^{\prime}-\lambda\right)$. Since $P$ or $Q$ has infinite rank, there exists a sequence $\left\{W_{j}\right\}_{j=1}^{\infty} \subset \mathcal{L}\left(Q \mathscr{H}_{2}, P \mathscr{H}_{1}\right)$ of finite rank operators such that $\left\{V_{i}\right\} \cup\left\{W_{j}\right\}$ is independent. For $S \in J$, let $[S]$ denote the image of $S$ in $J / \operatorname{Ran}\left(R_{J}^{\prime}-\lambda\right)$; note that $\left\{\left[M\left(W_{j}\right)\right]\right\}_{j=1}^{\infty}$ is independent. Indeed, if $c_{1}, \ldots, c_{m}$ are scalars such that $\sum c_{j}\left[M\left(W_{j}\right)\right]=0$, then $0=\left[M\left(\sum c_{j} W_{j}\right)\right]$, so $M\left(\sum c_{j} W_{j}\right) \in \mathcal{M} \cap \operatorname{Ran}\left(R_{J}^{\prime}-\lambda\right)$. Thus there exist scalars $d_{1}, \ldots, d_{n}$ such that $\sum c_{j} W_{j}=\sum d_{i} V_{i}$, whence each $c_{j}=0$. Since $\left\{\left[M\left(W_{j}\right)\right]\right\}_{j=1}^{\infty}$ is independent, $\operatorname{def}\left(R_{J}^{\prime}-\lambda\right)=\infty$, and thus $\lambda \in \sigma_{\mathrm{e}}\left(R_{J}^{\prime}\right)$. The case when $n=0$ is treated similarly; the proof is now complete.

LEMMA 2.13. $\sigma_{\mathrm{rr}}(A, B) \subset \sigma_{\mathrm{e}}\left(R_{J}(A, B)\right)$.

PROOF. By considerations with adjoints, the proof follows from Proposition 2.12 and the following observation: $R_{J}(A, B)-\lambda$ is semi-Fredholm if and only if $R_{J}\left(B^{*}, A^{*}\right)-\bar{\lambda}$ is semi-Fredholm, and in this case

$$
\operatorname{ind}\left(R_{J}(A, B)-\lambda\right)=\operatorname{ind}\left(R_{J}\left(B^{*}, A^{*}\right)-\bar{\lambda}\right)
$$

(see [11, Lemma 2.6]). 
Proof OF THEOREM 2.1. Let

$$
\sigma(A, B)=\sigma_{\mathrm{lr}}(A, B) \cup \sigma_{\mathrm{rl}}(A, B) \cup \sigma_{\mathrm{ll}}(A, B) \cup \sigma_{\mathrm{rr}}(A, B) .
$$

It follows from the definitions of Harte spectra and essential spectra that

$$
\sigma(A, B)=\sigma_{\mathrm{H}}(A) \circ \sigma_{\mathrm{He}}(B) \cup \sigma_{\mathrm{He}}(A) \circ \sigma_{\mathrm{H}}(B) .
$$

The inclusion $\sigma(A, B) \subset \sigma_{\mathrm{e}}\left(R_{J}\right)$ thus follows from Propositions 2.8, 2.10, and 2.12 and from Lemma 2.13. The reverse inclusion follows from (2.3).

3. Isolated points of the Harte joint spectrum. Recall the Riesz Decomposition Theorem [23, Theorem, p. 421]: If $A \in \mathcal{L}(\mathcal{H})$ and $\sigma(A)=\sigma_{1} \cup \sigma_{2}$ where $\sigma_{1}$ and $\sigma_{2}$ are disjoint nonempty closed subsets of $\sigma(A)$, then there exist complementary closed $A$-invariant subspaces $\mathcal{M}, \mathcal{N} \subset$ H such that $\sigma(A \mid \mathcal{M})=\sigma_{1}$ and $\sigma(A \mid \mathcal{N})=\sigma_{2} ;$ equivalently, $A$ is similar to an orthogonal direct sum $A_{1} \oplus A_{2}$, where $\sigma\left(A_{i}\right)=\sigma_{i}(i=1,2)$; finally, if $\sigma_{1}=\{\lambda\}$, a singleton, then $\lambda \in \sigma_{\mathrm{e}}(A)$ if and only if $\operatorname{dim} \mathcal{M}=\infty$. (This version of the Riesz Decomposition Theorem is discussed in [13].)

This decomposition depends on the existence of an analytic functional calculus for $A$ relative to $\sigma(A)$. If $A$ is a commutative $n$-tuple of operators, there is an analytic functional calculus for $A$ relative to $\sigma_{\mathrm{T}}(A)$, so the conclusions of the Riesz Decomposition Theorem carry over to this setting [26]. On the other hand, the functional calculus for $n$-tuples relative to $\sigma_{\mathrm{H}}(A)$ concerns only polynomials $[18$, 19], so it is not surprising that (as we show below) the spectral decomposition property fails for isolated points of Harte spectra.

If $\sigma$ is an isolated subset of $\sigma_{\mathrm{H}}(A)$ for which there is a corresponding spectral decomposition, we say that $\sigma$ is removable. If an isolated subset $\sigma$ of $\sigma_{\mathrm{H}}(A)$ is not removable, then $\sigma$ cannot be isolated in $\sigma_{\mathrm{T}}(A)$; thus the description of the isolated points of $\sigma_{\mathrm{H}}(A)$ provides data that is useful in computing $\sigma_{\mathrm{T}}(A) \backslash \sigma_{\mathrm{H}}(A)$. A computationally useful description of $\sigma_{\mathrm{T}}(A) \backslash \sigma_{\mathrm{H}}(A)$ is unknown even for $n=2$ (except in special cases, see $[4,5]$ ), and in the sequel we state our results only for pairs of operators; several of the results do, however, admit extensions to $n$-tuples.

The results of this section are also motivated by the following considerations concerning elementary operators. Let $n=1, A=\left(A_{1}\right), B=\left(B_{1}\right)$, or let $n=$ $2, A=\left(A_{1},-1\right), B=\left(1, B_{2}\right)$. The corresponding elementary operator $R_{A B}$ satisfies the following property:

$$
\begin{aligned}
& \text { If } \lambda \text { is in } \sigma(R) \backslash \sigma_{\mathrm{e}}(R) \text {, then } \lambda=\alpha \circ \beta \text {, where } \alpha \in \sigma_{\mathrm{H}}(A) \backslash \sigma_{\mathrm{He}}(A), \beta \in \\
& \sigma_{\mathrm{H}}(B) \backslash \sigma_{\mathrm{He}}(B) \text {, and } \alpha \text { is isolated in } \sigma_{\mathrm{H}}(A) \text { or } \beta \text { is isolated in } \sigma_{\mathrm{H}}(B)
\end{aligned}
$$

(see $[10,11])$. In the second example above, since $\alpha=\left(\alpha_{1}, \alpha_{2}\right)$ is isolated in $\sigma_{\mathrm{H}}(A)$ if and only if $\alpha_{1}$ is isolated in $\sigma\left(A_{1}\right)$ and $\alpha_{2}=-1$ (and similarly for $\beta=\left(\beta_{1}, \beta_{2}\right)$ and $\sigma\left(B_{2}\right)$ ), an argument based on the Riesz Decomposition Theorem allows one to compute ind $(R-\lambda)$ in terms of invariants associated with the $A_{i}$ 's and $B_{i}$ 's [10]. An analogous argument holds for the $n=1$ case [11].

For arbitrary elementary operators (indeed, even for $n=2$ ), we do not know whether $(*)$ is valid; if $(*)$ does hold, the lack of a spectral decomposition for arbitrary isolated points of $\sigma_{\mathrm{H}}(A) \backslash \sigma_{\mathrm{He}}(A)$ may introduce a further difficulty in computing ind $(R-\lambda)$. Of course, it may happen that the analogue of $(*)$ for 
Taylor spectra is valid (as in the above examples), in which case the Taylor version of the spectral decomposition theorem can be applied.

In the sequel $A$ and $B$ are commuting operators on a separable Hilbert space $\nVdash$. In the following example we show that, in general, there is little one can say about the location of $\alpha \in \sigma(A)$ and $\beta \in \sigma(B)$ in cases for which $(\alpha, \beta)$ is isolated in $\sigma_{\mathrm{H}}(A, B)$.

EXAMPLE 3.1. We will exhibit commuting positive operators $A$ and $B$ with $\sigma(A)=\sigma_{\mathrm{e}}(A)=\sigma(B)=\sigma_{\mathrm{e}}(B)=[0,1]$ such that $\sigma_{\mathrm{H}}(A, B) \backslash \sigma_{\mathrm{He}}(A, B)$ contains a sequence of isolated points of $\sigma_{\mathrm{H}}(A, B)$ that is dense in $\sigma_{\mathrm{H}}(A, B)$.

Let $\left\{I_{n}\right\}_{n=1}^{\infty}$ denote the sequence of "middle third" open intervals used to define the Cantor set. For $n \geq 1$, let $\mathscr{H}_{n}$ denote a copy of $\not{H}$, let $\left\{e_{i n}\right\}_{i=1}^{\infty}$ denote an orthonormal basis for $\mathfrak{k}_{n}$, and let $S_{n}=\left\{r_{i n}\right\}_{i=1}^{\infty}$ denote an enumeration of the distinct rational numbers in $I_{n}$. Let $S=\bigcup_{n=1}^{\infty} S_{n}$ and let $\left\{s_{i n}\right\}_{i, n=1}^{\infty}$ denote an enumeration of the distinct points of $S$ such that $s_{i n}$ is in $I_{i}$ (e.g., let $s_{i n}=r_{n i}$ for $i, n \geq 1)$. Define operators $A$ and $B$ on $\nvdash_{\infty}=\sum_{i=1}^{\infty} \bigoplus \nvdash_{n}$ as follows:

$$
A e_{i n}=r_{i n} e_{i n}, \quad B e_{i n}=s_{i n} e_{i n}, \quad i, n=1,2, \ldots
$$

Clearly $A$ and $B$ are commuting positive operators with $\sigma(A)=\sigma_{\mathrm{e}}(A)=\sigma(B)=$ $\sigma_{\mathrm{e}}(B)=[0,1]$. If $i, n \geq 1$, then $\left(r_{i n}, s_{i n}\right) \in \sigma_{\mathrm{p}}(A, B)$ (with joint eigenvector $\left.e_{i n}\right)$; we show that each $\left(r_{i n}, s_{i n}\right)$ is an isolated point of $\sigma_{\mathrm{H}}(A, B)$ belonging to $\sigma_{\mathrm{H}}(A, B) \backslash \sigma_{\mathrm{He}}(A, B)$ and $\left\{\left(r_{i n}, s_{i n}\right)\right\}_{i, n=1}^{\infty}$ is dense in $\sigma_{\mathrm{H}}(A, B)$.

To this end, recall that since $A$ and $B$ are normal, $\sigma_{\mathrm{H}}(A, B)=\sigma_{\mathrm{l}}(A, B)$ and $\sigma_{\mathrm{He}}(A, B)=\sigma_{\mathrm{le}}(A, B)\left[4\right.$, Corollary 3.9]. Fix $i_{0}, n_{0} \geq 1$. Suppose $(r, s) \in \sigma_{\mathrm{l}}(A, B)$ and let $\left\{h_{k}\right\}$ denote a sequence of unit vectors such that

$$
\lim _{k}\left\|(A-r) h_{k}\right\|^{2}+\left\|(B-s) h_{k}\right\|^{2}=0 .
$$

Thus

$$
h_{k} \equiv \sum_{i, n \geq 1} a_{i n}^{(k)} e_{i n}=\sum_{n \neq n_{0}} \sum_{i=1}^{\infty} a_{i n}^{(k)} e_{i n}+a_{i_{0} n_{0}}^{(k)} e_{i_{0} n_{0}}+\sum_{i \neq i_{0}}^{\infty} a_{i n_{0}}^{(k)} e_{i n_{0}} .
$$

It follows that

$$
\begin{aligned}
d_{k} \equiv & \left\|(A-r) h_{k}\right\|^{2}+\left\|(B-s) h_{k}\right\|^{2} \\
= & \sum_{n \neq n_{0}} \sum_{i=1}^{\infty}\left|a_{i n}^{(k)}\right|^{2}\left(\left|r_{i n}-r\right|^{2}+\left|s_{i n}-s\right|^{2}\right) \\
& +\left|a_{i_{0} n_{0}}^{(k)}\right|^{2}\left(\left|r_{i_{0} n_{0}}-r\right|^{2}+\left|s_{i_{0} n_{0}}-s\right|^{2}\right) \\
& +\sum_{i \neq i_{0}}\left|a_{i n_{0}}^{(k)}\right|^{2}\left(\left|r_{i n_{0}}-r\right|^{2}+\left|s_{i n_{0}}-s\right|^{2}\right) .
\end{aligned}
$$

Note that $\left(r_{i_{0} n_{0}}, s_{i_{0} n_{0}}\right) \in I_{n_{0}} \times I_{i_{0}}$; we claim that if $(r, s) \in I_{n_{0}} \times I_{i_{0}}$, then $r=r_{i_{0} n_{0}}$ and $s=s_{i_{0} n_{0}}$. Indeed, if $(r, s) \in I_{n_{0}} \times I_{i_{0}}$, then $\delta_{1} \equiv \operatorname{dist}\left(s, \partial I_{i_{0}}\right)>0$, and $\left|s_{i n_{0}}-s\right| \geq \delta_{1}$ for $i \neq i_{0}$; similarly, since $r \in I_{n_{0}}$, then $\delta_{2} \equiv \operatorname{dist}\left(r, \partial I_{n_{0}}\right)>0$ and $\left|r_{i n}-r\right| \geq \delta_{2}$ for $n \neq n_{0}$. If $\delta_{3} \equiv \operatorname{dist}\left((r, s),\left(r_{i_{0} n_{0}}, s_{i_{0} n_{0}}\right)\right)>0$, then $\delta \equiv$ $\min \left(\delta_{1}, \delta_{2}, \delta_{3}\right)>0$, so (3.2) implies that

$$
d_{k} \geq \sum_{n \neq n_{0}} \sum_{i=1}^{\infty}\left|a_{i n}^{(k)}\right|^{2} \delta_{2}^{2}+\left|a_{i_{0} n_{0}}^{(k)}\right|^{2} \delta_{3}^{2}+\sum_{i \neq i_{0}}\left|a_{i n_{0}}^{(k)}\right|^{2} \delta_{1}^{2} \geq \delta^{2}>0
$$


this contradiction to (3.1) shows that each $\left(r_{i_{0} n_{0}}, s_{i_{0} n_{0}}\right)$ is isolated in $\sigma_{\mathrm{H}}(A, B)$.

Now suppose $r=r_{i_{0} n_{0}}, s=s_{i_{0} n_{0}}$, and $\delta=\min \left(\delta_{1}, \delta_{2}\right)$; (3.2) implies

$$
d_{k} \geq \sum_{(i, n) \neq\left(i_{0}, n_{0}\right)}\left|a_{i n}^{(k)}\right|^{2} \delta^{2}
$$

so (3.1) shows that

$$
\lim _{k} \sum_{(i, n) \neq\left(i_{0}, n_{0}\right)}\left|a_{i n}^{(k)}\right|^{2}=0,
$$

whence $\lim _{k}\left|a_{i_{0} n_{0}}^{(k)}\right|=1$. It follows that

$$
\lim _{k}||\left(h_{k}, h_{k+1}\right)|-| a_{i_{0} n_{0}}^{(k)} a_{i_{0} n_{0}}^{(k+1)}||=0
$$

so that $\lim _{k}\left|\left(h_{k}, h_{k+1}\right)\right|=1$. Thus $\left\{h_{k}\right\}$ cannot be an orthonormal sequence, so [8] implies that $\left(r_{i_{0} n_{0}}, s_{i_{0} n_{0}}\right) \notin \sigma_{\mathrm{le}}(A, B)$.

Finally, if $(r, s) \in \sigma_{\mathrm{H}}(A, B)$ and $\delta_{4} \equiv \inf _{i, n \geq 1} \operatorname{dist}\left((r, s),\left(r_{i}, s_{i}\right)\right)>0$, then $\inf _{k} d_{k} \geq \delta_{4}^{2}$, and this contradiction implies that $\left\{\left(r_{i n}, s_{i n}\right)\right\}_{i, n=1}^{\infty}$ is dense in $\sigma_{\mathrm{H}}(A, B)$.

In contrast to this example, we show below that if $(\alpha, \beta)$ is an isolated point of $\sigma_{\mathrm{H}}(A, B)$ arising from $\alpha \in \sigma(A) \backslash \sigma_{\mathrm{e}}(A)$, then there is a "regularity" constraint against $\alpha$ which helps us locate $\alpha$.

For $\alpha \in \mathbf{C}$, define the $\alpha$-cross section of $\sigma_{\mathrm{H}}(A, B)$ as follows:

$$
\sigma_{\alpha}(B)=\left\{\beta \in \mathbf{C}:(\alpha, \beta) \in \sigma_{\mathrm{H}}(A, B)\right\} ;
$$

clearly $\sigma_{\alpha}(B) \subset \sigma(B)$ and $\sigma_{\alpha}(B)=\emptyset$ if $\alpha \notin \sigma(A)$. The projection property of Harte spectra (contained in [18, Theorem 4.2]) implies that if $\alpha \in \sigma(A)$, then $\sigma_{\alpha}(B) \neq \emptyset$, and $\bigcup_{\alpha \in \sigma(A)} \sigma_{\alpha}(B)=\sigma(B)$. In what follows we employ certain refinements of the projection property. For $\alpha \in \mathbf{C}$, define

$$
\begin{aligned}
& \sigma_{\alpha, 1}(B)=\left\{\beta \in \mathbf{C}:(\alpha, \beta) \in \sigma_{\mathrm{l}}(A, B)\right\} \quad\left(\subset \sigma_{\mathrm{l}}(B)\right), \\
& \sigma_{\alpha, \mathrm{le}}(B)=\left\{\beta \in \mathbf{C}:(\alpha, \beta) \in \sigma_{\mathrm{le}}(A, B)\right\} \quad\left(\subset \sigma_{\mathrm{le}}(B)\right) ;
\end{aligned}
$$

define $\sigma_{\alpha, \mathrm{r}}(B)$ and $\sigma_{\alpha, \mathrm{re}}(B)$ for right and right essential spectra analogously.

For $\alpha \in \sigma(A)$, let $\mathcal{M}=\operatorname{ker}(A-\alpha)$ and $\mathcal{N}=\operatorname{ker}\left((A-\alpha)^{*}\right)$. Since $B$ commutes with $A, \mathcal{M}$ is $B$-invariant and $\mathcal{N}$ is $B^{*}$-invariant.

PROPOSITION 3.2. If $A-\alpha$ has closed range, then

(i) $\sigma_{\alpha, 1}(B)=\sigma_{1}(B \mid \mathcal{M})$;

(ii) $\sigma_{\alpha, \mathrm{le}}(B)=\sigma_{\mathrm{le}}(B \mid \mathcal{M})$ (when $\mathcal{M}$ is infinite dimensional);

(iii) $\sigma_{\alpha, \mathrm{r}}(B)=\sigma_{\mathrm{l}}\left(B^{*} \mid \mathcal{N}\right)^{*}\left(\right.$ for $\left.\sigma \subset \mathbf{C}, \sigma^{*}=\{\bar{\lambda}: \lambda \in \sigma\}\right)$;

(iv) $\sigma_{\alpha, \mathrm{re}}(B)=\sigma_{\mathrm{le}}\left(B^{*} \mid \mathcal{N}\right)^{*}$.

ProOF. (i) Let $\mathcal{M}^{\prime}=\mathcal{H} \ominus \mathcal{M}$; relative to the decomposition $\forall=\mathcal{M} \oplus \mathcal{M}^{\prime}$, the matrices of $A-\alpha$ and $B$ are of the form

$$
A-\alpha=\left(\begin{array}{cc}
0 & A_{12} \\
0 & A_{22}
\end{array}\right), \quad B=\left(\begin{array}{cc}
B_{11} & B_{12} \\
0 & B_{22}
\end{array}\right),
$$

and since $\operatorname{Ran}(A-\alpha)$ is closed,

$$
A_{12}^{*} A_{12}+A_{22}^{*} A_{22} \text { is invertible. }
$$


Now $B_{11}=B \mid \mathcal{M}$ and, clearly, $\sigma_{1}\left(B_{11}\right) \subset \sigma_{\alpha, 1}(B)$. Conversely, let $\beta \in \sigma_{\alpha, 1}(B)$ and let $\left\{z_{n}\right\} \subset \not$ be a sequence of unit vectors such that $\left\|(A-\alpha) z_{n}\right\|+\left\|(B-\beta) z_{n}\right\| \rightarrow 0$. Let $z_{n}=x_{n}+y_{n}, x_{n} \in \mathcal{M}, y_{n} \in \mathcal{M}^{\prime}$. Since $\left\|(A-\alpha) z_{n}\right\| \rightarrow 0,(* *)$ implies $\left\|y_{n}\right\| \rightarrow 0$, whence $\left\|\left(B_{11}-\beta\right) x_{n}\right\| \rightarrow 0$ and $\left\|x_{n}\right\| \rightarrow 1$. Thus $\beta \in \sigma_{1}(B \mid \mathcal{M})$; this proves (i).

(ii) Assume $\mathcal{M}$ is infinite dimensional; the inclusion $\sigma_{\text {le }}(B \mid \mathcal{M}) \subset \sigma_{\alpha, \text { le }}(B)$ is clear. Conversely, suppose $\beta \in \sigma_{\alpha, \text { le }}(B)$. There exists an infinite rank orthogonal projection

$$
P=\left(\begin{array}{ll}
P_{11} & P_{12} \\
P_{21} & P_{22}
\end{array}\right)
$$

such that $(A-\alpha) P,(B-\beta) P \in \mathcal{K}(\mathcal{H})$. Since $(A-\alpha) P$ is compact, (**) implies that $P_{21}, P_{22} \in \mathcal{K}(\mathcal{H})$. Since $P_{12}=P_{11} P_{12}+P_{12} P_{22}$ and $P$ is not compact, it follows that $P_{11}$ is not compact. Thus $\tilde{P}_{11}$ is a nonzero projection such that $\left(\tilde{B}_{11}-\beta\right) \tilde{P}_{11}=0$ and thus $\beta \in \sigma_{\text {le }}(B \mid \mathcal{M})$.

(iii) and (iv) follow from (i) and (ii) by taking adjoints.

For the case when $\operatorname{Ran}(A-\alpha)$ is not closed, we obtain analogues of Proposition $3.2(\mathrm{ii})$, (iv) as follows.

For $A \in \mathcal{L}(\mathcal{H})$ and $\alpha \in \mathbf{C}$, let $J \equiv J(A, \alpha)=\{Q \in \mathcal{L}(\mathcal{H}):(A-\alpha) Q \in \mathcal{K}(\not{H})\} ; J$ is a closed right ideal of $\mathcal{L}(\mathcal{K})$ and $\mathcal{K}(\mathcal{H}) \subset J$; clearly, $J=\mathcal{L}(\mathcal{K})$ if and only if $A-\alpha \in \mathcal{K}(\not{K})$, and $\mathcal{J}=\mathcal{K}(\not{H})$ if and only if $\alpha \notin \sigma_{\mathrm{le}}(A)$. If $B \in \mathcal{L}(\not{K})$ and $B A-A B \in \mathcal{K}(\mathcal{H})$, then $B J \subset J$. In this case, let $\tilde{\mathcal{L}}=\mathcal{L}(\mathcal{H}) / \mathcal{J}$ and define $L_{\tilde{B}}: \tilde{\mathcal{L}} \rightarrow \tilde{\mathcal{L}}$ by $L_{\tilde{B}}(\tilde{Q})=\tilde{B} \tilde{Q} ;$ thus $L_{\tilde{B}} \in \mathcal{L}(\tilde{\mathcal{L}})$.

Proposition 3.3. (i) If $\alpha \in \sigma_{\mathrm{le}}(A)$ (in particular, if $\operatorname{Ran}(A-\alpha)$ is not closed), then $\sigma_{\alpha, \mathrm{le}}(B)=\sigma_{\pi}\left(L_{\tilde{B}}\right)=\sigma_{\mathrm{p}}\left(L_{\tilde{B}}\right)\left(\sigma_{\pi}(\cdot)\right.$ and $\sigma_{\mathrm{p}}(\cdot)$ denote approximate point and point spectra).

(ii) If $\alpha \in \sigma_{\mathrm{re}}(A)$, then $\sigma_{\alpha, \mathrm{re}}(B)=\sigma_{\pi}\left(L_{\tilde{B}^{*}}\right)^{*}=\sigma_{\mathrm{p}}\left(L_{B^{*}}\right)^{*}$ (relative to $\mathrm{J}=$ $J\left(A^{*}, \bar{\alpha}\right)$.

ProOF. (i) Let $\beta \in \sigma_{\pi}\left(L_{\tilde{B}}\right)$. Then there exists a sequence $\left\{\tilde{Q}_{n}\right\} \subset \tilde{\mathcal{L}},\left\|\tilde{Q}_{n}\right\|=1$, such that $\left\|\left(L_{\tilde{B}}-\beta\right)\left(\tilde{Q}_{n}\right)\right\| \rightarrow 0$, i.e., $\left\|(\tilde{B}-\beta) \tilde{Q}_{n}\right\| \rightarrow 0$. Suppose there exist operators $S, T \in \mathcal{L}(\mathcal{H})$ such that $\tilde{S}(\tilde{A}-\alpha)+\tilde{T}(\tilde{B}-\beta)=1$. Since $(\tilde{A}-\alpha) \tilde{Q}_{n}=0$ and $\left\|(\tilde{B}-\beta) \tilde{Q}_{n}\right\| \rightarrow 0$, then

$$
\begin{aligned}
\left\|\tilde{Q}_{n}\right\| & =\left\|\tilde{S}(\tilde{A}-\alpha) \tilde{Q}_{n}+\tilde{T}(\tilde{B}-\beta) \tilde{Q}_{n}\right\|=\left\|\tilde{T}(\tilde{B}-\beta) \tilde{Q}_{n}\right\| \\
& \leq\|\tilde{T}\|\left\|(\tilde{B}-\beta) \tilde{Q}_{n}\right\| \rightarrow 0,
\end{aligned}
$$

and this contradiction shows that $(\alpha, \beta) \in \sigma_{\mathrm{le}}(A, B)$. Conversely, if $(\alpha, \beta) \in$ $\sigma_{\mathrm{le}}(A, B)$, then there exists an infinite rank projection $P$ such that $(A-\alpha) P \in \mathcal{K}(\mathscr{K})$ and $\left(L_{\tilde{B}}-\beta\right)(\tilde{P})=0$; thus $\beta \in \sigma_{\mathrm{p}}\left(L_{\tilde{B}}\right)$, and the proof is complete. (The preceding argument has features in common with the proof of [18, Theorem 4.2].)

(ii) Take adjoints and apply (i).

We next recall some concepts studied by $C$. Apostol [1]. For $T \in \mathcal{L}(\not{K})$ and $\lambda \in \rho_{\mathrm{SF}}(T)$, let min.ind. $(T-\lambda)=\min \{\operatorname{nul}(T-\lambda), \operatorname{def}(T-\lambda)\} ; \lambda$ is a regular point of $\rho_{\mathrm{SF}}(T)$ if $\alpha \rightarrow P_{\operatorname{ker}(T-\alpha)}$ is (norm) continuous at $\alpha=\lambda$, otherwise, $\lambda$ is a singular point of $\rho_{\mathrm{SF}}(T)$. Let $\rho_{\mathrm{SF}}^{\mathrm{r}}(T)$ and $\rho_{\mathrm{SF}}^{\mathrm{s}}(T)$ denote, respectively, the sets of all regular and singular points of $\rho_{\mathrm{SF}}(T)$. We denote the regular and singular points of $\sigma(T) \backslash \sigma_{\mathrm{e}}(T)$ by $\rho_{\mathrm{F}}^{\mathrm{r}}(T)$ and $\rho_{\mathrm{F}}^{\mathrm{s}}(T)$. In [1, Theorem 2.2] C. Apostol proved 
that, for $T$ in $\mathcal{L}(\not \nvdash), \rho_{\mathrm{SF}}^{\mathrm{r}}(T)$ is an open set, $\rho_{\mathrm{SF}}^{\mathrm{r}}(T)=\rho_{\mathrm{SF}}^{\mathrm{r}}\left(T^{*}\right)^{*}$, and $\rho_{\mathrm{SF}}^{\mathrm{s}}(T)$ is at most a denumerable set and has no accumulation points in $\rho_{\mathrm{SF}}(T)$.

Proposition 3.4. Let $A$ and $B$ be commuting operators in $\mathcal{L}(\mathcal{H})$. If $\alpha \in$ $\rho_{\mathrm{F}}(A) \equiv \mathbf{C} \backslash \sigma_{\mathrm{e}}(A)$ and $(\alpha, \beta)$ is isolated in $\sigma_{\mathrm{H}}(A, B)$, then $\alpha \in \rho_{\mathrm{F}}^{\mathrm{s}}(A)$.

ProOF. Suppose, to the contrary, that $\alpha \in \rho_{\mathrm{F}}^{\mathrm{r}}(A)$. Let $\mathcal{M}=\operatorname{ker}(A-\alpha)$ and $\mathcal{N}=\operatorname{ker}\left((A-\alpha)^{*}\right)$. Since $\mathcal{M}$ and $\mathcal{N}$ are finite dimensional, Proposition 3.2 implies that $\beta \in \sigma_{\alpha}(B)=\sigma_{\alpha, 1}(B) \cup \sigma_{\alpha, \mathrm{r}}(B)=\sigma(B \mid \mathcal{M}) \cup \sigma\left(B^{*} \mid \mathcal{N}\right)^{*}$. Suppose $\beta \in \sigma(B \mid \mathcal{M})$ (so, in particular, $\mathcal{M} \neq\{0\}$ ). Since $\alpha$ is regular (and $\rho_{\mathrm{F}}^{\mathrm{r}}(A)$ is open), there exists a sequence $\left\{\alpha_{n}\right\}$ of distinct regular points of $\rho_{\mathrm{F}}(A)$ such that $\alpha_{n} \rightarrow \alpha$. Let $P_{n}=P_{\operatorname{ker}\left(A-\alpha_{n}\right)}$ and $P=P_{\operatorname{ker}(A-\alpha)}$; let $k=2\|B\|$. Since $\alpha$ is regular,

$$
\left\|\left(B P_{n}+k\left(1-P_{n}\right)\right)-(B P+k(1-P))\right\| \rightarrow 0 .
$$

Now $\beta$ is an isolated point of $\sigma(B P+k(1-P)$ ) (which is finite), so by upper semicontinuity of the separate parts of the spectrum [20, Corollary 1.6], given $m>4 / k$, there exists $n_{m}>m$ such that

$$
\sigma\left(B P_{n_{m}}+k\left(1-P_{n_{m}}\right)\right) \cap\{z:|z-\beta|<1 / m\} \neq \emptyset .
$$

(We may also assume that $n_{m}>n_{m-1}$.) Thus there exists

$$
\beta_{m} \in \sigma\left(B P_{n_{m}}+k\left(1-P_{n_{m}}\right)\right)
$$

such that $\left|\beta_{m}-\beta\right|<1 / m$. Since $\left|\beta_{m}\right|<|\beta|+1 / m<\|B\|+k / 4=\frac{3}{4} k$, then $\beta_{m} \neq k$, and thus $\beta_{m} \in \sigma\left(B P_{n_{m}} \mid P_{n_{m}} \not \mathcal{H}\right) \subset \sigma_{\alpha_{n_{m}, 1}}(B)$ (Proposition 3.2(i)). Thus $\left\{\left(\alpha_{n_{m}}, \beta_{m}\right)\right\}_{m}$ is a sequence of distinct points of $\sigma_{\mathrm{H}}(A, B)$ convergent to $(\alpha, \beta)$. This contradiction shows that $\alpha$ is singular. In the case when $\beta \in \sigma_{\alpha, \mathbf{r}}(B)$, a similar argument works since the function $\lambda^{\prime} \rightarrow P_{\operatorname{ker}\left(A-\lambda^{\prime}\right)^{*}}$ is continuous at $\alpha$.

REMARK. R. Curto [6] has proved a result which implies that, if $\alpha \in \rho_{\mathrm{F}}(A)$ and $(\alpha, \beta)$ is isolated in $\sigma_{\mathrm{H}}(A, B)$, then $(\alpha, \beta)$ is isolated in $\sigma_{\mathrm{T}}(A, B)$, whence $(\alpha, \beta)$ is removable. Our concluding example shows that neither this result nor Proposition 3.4 can be extended to the case when $\alpha \in \rho_{\mathrm{SF}}(A)$.

EXAMPLE 3.5. Let $U \in \mathcal{L}(\not H)$ denote the unilateral shift of multiplicity one. Let $\mathfrak{H}_{\infty}=\mathfrak{H} \oplus \mathfrak{H} \oplus \mathfrak{H} \oplus \cdots$, and define operators $A, B \in \mathcal{L}\left(\mathfrak{H}_{\infty}\right)$ by the operator matrices

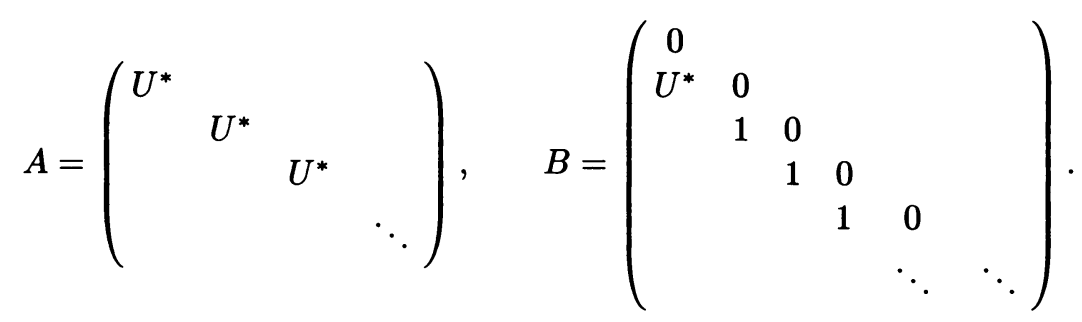

Straightforward matrix calculations show that $A$ and $B$ commute and that no nontrivial idempotent commutes with both $A$ and $B$. Well-known properties of the shift imply that $0 \in \rho_{\mathrm{SF}}^{\mathrm{r}}(A)$; we will show that $(0,0)$ is an isolated point of $\sigma_{\mathrm{H}}(A, B)$ and that $(0,0) \notin \sigma_{\mathrm{He}}(A, B)$. Since no proper idempotent commutes wiht both $A$ and $B,(0,0)$ is necessarily nonremovable. Thus the results of $[6]$ and Proposition 3.4 cannot be extended to the case when $\alpha \in \rho_{\mathrm{SF}}(A)$. 
Let $\alpha=0$. A calculation shows that the matrix of $B \mid \operatorname{ker}(A)$ is unitarily equivalent to $0_{\mathbf{C}^{1}} \oplus U$, so Proposition 3.2(i) implies that $\sigma_{\alpha, 1}(B)=\sigma_{1}(B \mid \operatorname{ker}(A))=\{0\} \cup \mathbf{T}$ (where $\mathbf{T}$ denotes the unit circle). Since $\operatorname{ker}\left(A^{*}\right)=\{0\}$, Proposition 3.2 implies that $\sigma_{\alpha}(B)=\{0\} \cup \mathbf{T}$. Let $0<|\alpha|<1$. A calculation shows that $B \mid \operatorname{ker}(A-\alpha)$ is unitarily equivalent to the unilateral weighted shift with weights $\alpha, 1,1,1, \ldots$, and thus $B \mid \operatorname{ker}(A-\alpha)$ is similar to $U[\mathbf{1 7}$, p. 47]. Now Proposition 3.2 implies that $\sigma_{\alpha}(B)=\sigma_{\alpha, 1}(B)=\sigma_{1}(U)=\mathbf{T}$. Thus $(0,0)$ is an isolated point of $\sigma_{\mathrm{H}}(A, B)$. Since $A^{*} A+B^{*} B$ and $A A^{*}+B B^{*}$ are Fredholm, it follows from Lemma 2.4 that $(0,0) \notin \sigma_{\mathrm{He}}(A, B)$.

Finally, note that since $(0,0)$ is nonremovable relative to $\sigma_{\mathrm{H}}(A, B)$, it cannot be isolated in $\sigma_{\mathrm{T}}(A, B)$. Indeed, let $\mathbf{D}$ denote the closed unit disk; then $\sigma_{\mathrm{H}}(A, B)=$ $\{(0,0)\} \cup \operatorname{bdry}(\mathbf{D} \times \mathbf{D})$, and $\sigma_{\mathrm{T}}(A, B)=\mathbf{D} \times \mathbf{D}$.

REMARK. After submitting this paper for publication we learned of the following developments:

(i) R. Curto [7] has recently studied relationships between Harte and Taylor spectra and in so doing has found a computational method for determining points of $\sigma_{\mathrm{T}}(A) \backslash \sigma_{\mathrm{H}}(A)$ for $n=2$ (cf. the introductory remarks of $\S 3$ ). Moreover, he has found proofs of (1.7) and the identity

$$
\sigma_{\mathrm{He}}(A) \circ \sigma_{\mathrm{H}}(B) \cup \sigma_{\mathrm{H}}(A) \circ \sigma_{\mathrm{He}}(B)=\sigma_{\mathrm{Te}}(A) \circ \sigma_{\mathrm{T}}(B) \cup \sigma_{\mathrm{T}}(A) \circ \sigma_{\mathrm{Te}}(B)
$$

that are independent of elementary operators [7, Theorem 3.14 and Corollary 3.16] (cf. the remark following (1.7)). When combined with Theorem 2.1, the latter identity yields the following analogue of (1.2) for essential spectra [7, Corollary 3.17]:

$$
\sigma_{\mathrm{e}}\left(R_{J}\right)=\sigma_{\mathrm{Te}}(A) \circ \sigma_{\mathrm{T}}(B) \cup \sigma_{\mathrm{T}}(A) \circ \sigma_{\mathrm{Te}}(B) .
$$

In [7, Example 4.1], Curto studies a generalization of Example 3.2.

(ii) In a forthcoming sequel to this note [29] we study the problem of computing $\operatorname{ind}\left(R_{J}-\lambda\right)$. For $\lambda \in \sigma\left(R_{J}\right) \backslash \sigma_{\mathrm{e}}\left(R_{J}\right)$, let

$$
X_{\lambda}=\left\{(\alpha, \beta) \in \sigma_{\mathrm{T}}(A) \times \sigma_{\mathrm{T}}(B): \alpha \circ \beta=\lambda\right\} .
$$

Using (1.7) and (3.3) we compute ind $\left(R_{J}-\lambda\right)$ under the following hypothesis: $X_{\lambda}$ is finite and, for each $(\alpha, \beta)$ in $X_{\lambda}$, either $\alpha$ is isolated in $\sigma_{\mathrm{T}}(A)$ or $\beta$ is isolated in $\sigma_{\mathrm{T}}(B)$. (See the introductory remarks of $\S 3$.)

(iii) A revised version of $[\mathbf{3}]$ reflects some changes from an earlier version to which we have referred in the preceding text. For an operator $T$ on a Banach space $\mathcal{X}$ let

$$
\sigma_{\mathrm{e}}^{+}(T)=\{\lambda \in \mathbf{C}: \operatorname{Ran}(T-\lambda) \text { is not closed or } \operatorname{nul}(T-\lambda)=\infty\}
$$

and

$$
\sigma_{\mathrm{e}}^{-}(T)=\{\lambda \in \mathbf{C}: \operatorname{Ran}(T-\lambda) \text { is not closed or } \operatorname{def}(T-\lambda)=\infty\} ;
$$

thus $\sigma_{\mathrm{e}}^{+}(T) \subset \sigma_{\mathrm{le}}(T)$ and $\sigma_{\mathrm{e}}^{-}(T) \subset \sigma_{\mathrm{re}}(T)$ [28, Theorem 4]. In the version of [3] that we have used above, the authors prove that $\sigma_{\mathrm{le}}\left(R_{A B}\right)=\sigma_{\mathrm{lr}}$, while in the revised version they prove $\sigma_{\mathrm{e}}^{+}\left(R_{A B}\right)=\sigma_{\mathrm{lr}}$. However, the proof of the latter identity still shows that $\sigma_{\mathrm{le}}(R) \subset \sigma_{\mathrm{lr}}$, so [28, Theorem 4] implies that $\sigma_{\mathrm{le}}(R)=\sigma_{\mathrm{lr}}$, as required for (1.10). Similarly, $\sigma_{\mathrm{re}}(R)=\sigma_{\mathrm{e}}^{-}(R)=\sigma_{\mathrm{rl}}$. A corresponding argument with $R_{J}$ can be used to establish (2.1) $-(2.3)$ from the revised version of $[3]$. 


\section{REFERENCES}

1. C. Apostol, The correction by compact perturbation of the singular behavior of operators, Rev. Roumaine Math. Pures Appl. 21 (1976), 155-175.

2. __ Inner derivations with closed range, Rev. Roumaine Math. Pures Appl. 21 (1976), $249-265$.

3. A. Carrillo and C. Hernández, Spectra of constructs of a system of operators, Proc. Amer. Math. Soc. 91 (1984), 426-433.

4. R. Curto, Fredholm and invertible $n$-tuples of operators. The deformation problem, Trans. Amer. Math. Soc. 266 (1981), 129-159.

5. _ The spectra of elementary operators, Indiana Univ. Math. J. 32 (1983), 193-197.

6. _ Private communication.

7. Connections between Harte and Taylor spectra, Preprint.

8. A. T. Dash, Joint essential spectra, Pacific J. Math. 64 (1976), 119-128.

9. L. A. Fialkow, Elements of spectral theory for generalized derivations, J. Operator Theory 3 (1980), 89-113.

10. __ Elements of spectral theory for generalized derivations. II: The semi-Fredholm domain, Canad. J. Math. 33 (1981), 1205-1231.

11. __ Essential spectra of elementary operators, Trans. Amer. Math. Soc. 267 (1981), 157-174.

12. __ Spectral properties of elementary operators, Acta Sci. Math. (Szeged) 46 (1983), 269-282.

13. L. A. Fialkow and D. A. Herrero, Inner derivations with closed range in the Calkin algebra, Indiana Univ. Math. J. 33 (1984), 185-211.

14. P. A. Fillmore, J. G. Stampfli and J. P. Williams, On the essential numerical range, the essential spectrum, and a problem of Halmos, Acta Sci. Math. (Szeged) 33 (1972), 179-192.

15. I. C. Gohberg and M. G. Krein, Introduction to the theory of linear nonselfadjoint operators, Transl. Math. Monographs, vol. 18, Amer. Math. Soc., Providence, R.I., 1969.

16. D. W. Hadwin, An asymptotic double commutant theorem for $C^{*}$-algebras, Trans. Amer. Math. Soc. 244 (1978), 273-297.

17. P. R. Halmos, A Hilbert space problem book, Van Nostrand, Princeton, N.J., 1967.

18. R. Harte, Spectral mapping theorems, Proc. Roy. Irish Acad. 72(A) (1972), 89-107.

19. __ Tensor products, multiplication operators and the spectral mapping theorem, Proc. Roy. Irish Acad. 73(A) (1973), 285-302.

20. D. A. Herrero, Approximation of Hilbert space operators. I, Research Notes in Math., Vol. 72, Pitman, Boston, 1983.

21. T. Kato, Perturbation theory for linear operators, Springer-Verlag, New York, 1966.

22. G. Lumer and M. Rosenblum, Linear operator equations, Proc. Amer. Math. Soc. 10 (1959), 32-41.

23. F. Riesz and B. Sz.-Nagy, Functional analysis, Ungar, New York, 1955.

24. R. Schatten, Norm ideals of completely continuous operators, Ergeb. Math. Grenzgeb., SpringerVerlag, Berlin and New York, 1960.

25. J. Taylor, A joint spectrum for several commuting operators, J. Funct. Anal. 6 (1970), 172-191.

26. _ The analytic functional calculus for several commuting operators, Acta Math. 125 (1970), 1-38.

27. D. Voiculescu, A noncommutative Weyl-von Neumann theorem, Rev. Roumaine Math. Pures Appl. 21 (1976), 97-113.

28. J. J. Buoni, R. Harte and T. Wickstead, Upper and lower Fredholm spectra, Proc. Amer. Math. Soc. 66 (1977), 309-314.

29. L. A. Fialkow, The index of an elementary operator, Preprint.

Department of Mathematics, State University of NeW York at NeW Paltz, NEW PALTZ, NEW YORK 12561 